\title{
Factores asociados al rendimiento de la comprensión lectora en estudiantes de secundaria
}

\section{Factors associated to public secondary school learners' reading comprehension}

\author{
DOI: https://doi.org/10.32870/dse.v0i23.695
}

\author{
Magally G. Sánchez-Domínguez* \\ Jesús Izquierdo**
}

\begin{abstract}
Resumen
Esta investigación indagó los factores académicos, socioculturales y personales de estudiantes de secundaria con perfil óptimo o endeble en comprensión lectora. Para determinar tales perfiles, de 151 estudiantes de tercer año en una escuela pública del sureste mexicano, se seleccionaron estudiantes cuya puntuación fluctuaba con una desviación estándar por debajo $(n=40)$ o por arriba $(n=29)$ de la media grupal en una prueba estandarizada. Los factores académicos, socioculturales y personales se exploraron empleando un cuestionario con 77 preguntas. Los datos en la encuesta revelan ciertas similitudes entre ambos tipos de estudiantes. Por ejemplo, todos leen por gusto o recomendación familiar y emplean la tecnología durante la lectura. No obstante, también muestran diferencias. Los estudiantes con perfil óptimo leen para mejorar su expresión, pero los participantes con perfil endeble leen para proyectar una imagen positiva. Partiendo de diversas reflexiones, se presentan recomendaciones para replicar el estudio con otras poblaciones.
\end{abstract}

Palabras clave: Comprensión lectora - educación secundaria - factores de la lectura - prueba de lectura.

\begin{abstract}
This study examined the academic, sociocultural and personal conditions of secondary-school students with different reading comprehension profiles. A standardized test and a survey were administered to 151 students from a public school in the Southeast of Mexico. Using the participants' score, the group's mean and group's standard deviation in the test's reading section, the learners were classified with optimal ( $n$ $=29)$ or weak $(n=40)$ reading profiles. The survey also explored the academic, sociocultural and personal conditions through 77 questions. The frequency analyses of the survey answers revealed similarities between both student types; for instance, they all read for pleasure or following family recommendations and used technology as they read. Nevertheless, diverging results also emerged. Learners with optimal
\end{abstract}

* Doctora en Educación. Líneas de investigación y docencia: comprensión lectora en educación básica: secundaria, los factores asociados a ella y las tecnologías de la información y comunicación tecnológicas en la comprensión lectora; estudios con diseño cuasiexperimental. Integrante del Sistema Estatal de Investigadores por el Consejo de Ciencia y Tecnología del Estado de Tabasco. México. isoldemp@hotmail.com ** Doctor en Educación en Segundas Lenguas. Profesor-Investigador Titular de la Universidad Juárez Autónoma de Tabasco, México. jesus.izquierdo@mail.mcgill.ca 
reading profiles read to improve their expression, whereas learners with weak reading profiles read to project a positive image. Finally, recommendations are put forward to replicate the study with other learner populations.

Key words: Reading comprehension - secondary education - reading conditions - reading test.

\section{Introducción}

La lectura es una valiosa herramienta de aprendizaje que se transforma en conocimientos de relevancia para el ámbito educativo y el desarrollo del intelecto humano (Cassany, Luna, Sanz, 2003; Delors, 1998; Solé, 2012). A pesar de la importancia de la lectura, para los investigadores de los años cincuenta, la lectura y la comprensión lectora fueron motivo de debate y preocupación por las limitaciones en torno a la generación de conocimientos en pro de la educación (Monroy, Gómez, 2009). Para la década de los setenta, ante la necesidad de hacer aportaciones científicas concernientes a la comprensión lectora, diversos estudiosos valoraron las razones que incidían en el desempeño lector, y analizaron cómo se desarrollaba el proceso lector de los estudiantes (Monroy, Gómez, 2009; González, Barba, González, 2010).

Al ser la comprensión lectora un área crucial en el desarrollo del individuo, que implica cierto grado de complejidad (González et al., 2010; Kendeou, McMaster, Christ, 2016; Márquez, 2017; Solé, 2012), instancias como la Organización para la Cooperación y el Desarrollo Económicos ([OCDE] 2016a) y la Organización de las Naciones Unidas para la Educación, la Ciencia y la Cultura, (UNESCO por sus siglas en inglés) (2016), han desarrollado políticas educativas con la intención de propiciar la mejora de la competencia lectora de los educandos. No obstante, pese a los esfuerzos de diversas instituciones educativas para cumplir con estas políticas, desde la educación primaria hasta la formación terciaria aún hay mucho por hacer en el fortalecimiento del desempeño de la comprensión lectora. En el caso de los estudiantes de nivel secundaria, en las instituciones educativas se observa un laxo aprovechamiento en el desarrollo de la comprensión lectora (González et al., 2010). Para el Institute for Statistics (UIS, 2017a) de la UNESCO, los datos en el desempeño de la comprensión lectora en estos estudiantes son desalentadores. A nivel mundial, 230 millones de jóvenes de secundaria no logran los conocimientos mínimos indispensables de la competencia lectora. De estos, 19 millones son estudiantes de América Latina; seis de cada diez adolescentes no alcanzan los niveles mínimos de comprensión lectora al concluir la educación secundaria (UIS, 2017a; 2017b).

Otro panorama que confirma la necesidad de volcar esfuerzos para promover el desarrollo en la comprensión lectora es el presentado por la Organización para la Cooperación y el Desarrollo Económicos (OCDE, 2016a) en su informe de 2015. En este reporte presenta los resultados de la prueba aplicada por el Programme for International Student Assessment (PISA) a los estudiantes cuando finalizan la educación secundaria. Esta prueba, generalmente referida como la

Diólo pos sobre Educación año 12 | número 23 | julio-diciembre 2021 | ISSN 2007-2171 
prueba PISA (véase Díaz-Barriga, 2015, Gracida, 2012; Madero, Gómez, 2013; Monroy, Gómez, 2009), comprende el aprovechamiento en lectura, matemáticas y ciencias. Afín al desempeño lector, de los 72 países asociados a la OCDE Singapur ocupó el primer lugar; Noruega fue líder en Europa; y en América Latina, Chile se mantuvo con notables resultados. En contraste, Uruguay, Costa Rica, Colombia, Brasil, Perú y República Dominicana no destacaron. En México, los datos acerca del aprovechamiento en la comprensión lectora casi siempre se sitúan debajo de la media estimada por la OCDE (2016b). Estos resultados generalmente coinciden con los alcanzados en la evaluación del Plan Nacional para la Evaluación de los Aprendizajes (Planea), realizada por el Instituto Nacional para la Evaluación de la Educación ${ }^{1}$ ([INEE] 2016). Sin duda, estos números evidencian que, independientemente de los esfuerzos de organismos e instituciones educativas, el desarrollo de la comprensión lectora representa una problemática real en los estudiantes de formación secundaria en América Latina, y principalmente en México.

Sobre la comprensión lectora, es vital reconocer los términos empleados por diversos autores, tales como comprensión de lectura (Johnston, Barnes, Desrochers, 2008; Martínez-Díaz, Díaz, Rodríguez, 2011; Zarzosa, Martínez, 2011), lectura de comprensión (Martínez-Díaz et al., 2011; Saulés, 2012), lectura comprensiva (Franco, Cárdenas, Santrich, 2016; Zarzosa, Martínez, 2011) y habilidades de comprensión lectora (Johnston et al., 2008; Pourhosein, Banou, 2016), entre otros. Para dar claridad al panorama de la comprensión lectora, resulta de interés considerar algunas acepciones. Jiménez (2014) conceptualiza la comprensión lectora como las habilidades intelectuales o emocionales desarrolladas por los individuos en la comprensión de un texto. Por su parte, el Reading Study Group (RAND por sus siglas en inglés, 2002) la define como "el proceso de extracción y construcción simultáneas de significado a través de la interacción y la participación con el lenguaje escrito" (p. 11). Hashemifardnia, Namaziandost y Sajad (2018) describen la comprensión lectora como "un proceso de pensamiento activo que depende no solo de la habilidad de comprensión sino también de la experiencia de los estudiantes" (p. 667). Finalmente, para Franco (2016) y González et al. (2010), el punto nodal de la comprensión de lectura es permitirle al lector construir sus propios significados emanados de un texto o de un autor. A partir de los planteamientos conceptuales anteriores, se deduce que la comprensión lectora es una de las actividades humanas más complejas (Cassany, 2004; Kendeou et al., 2016) a las que se enfrenta todo individuo, y comprende disímiles procesos.

Esos procesos de lectura, según el Ministerio de Educación del Perú (MEP, 2018), la UNESCO (2016), la OCDE $(2009,2013)$ y el INEE $(2016,2017)$ son: $a)$ extraer y recuperar, b) integrar e interpretar, c) reflexionar y evaluar. Al mismo tiempo, esos procesos implican niveles de logro y diversos grados de complejidad (Cassany et al., 2003; Gracida, 2012; INEE, 2016, 2017; Martínez-Díaz et al., 2011; OCDE, 2009, 2013; Saulés, 2012; Solé, 2012). Congruente con esta visión institucio-

1 Este organismo desapareció en 2018; posteriormente, se creó el Instituto Nacional para la Revalorización del Magisterio y la Mejora Continua de la Educación, asumiendo las mismas funciones del INEE, pero dejando fuera las evaluaciones a los docentes. 
nal, diferentes teóricos coinciden en que la comprensión lectora integra procesos discrepantes. Franco et al. (2016) señalan los procesos de conceptualización, análisis, generalización, interpretación y síntesis. Coromoto y Gutiérrez (2012) los categorizan como el análisis y la interpretación. Johnston et al. (2008) refieren la existencia de disímiles habilidades pero solo señalan la inferencia. Avendaño y Martínez (2013) sugieren la existencia de procesos con distintos niveles de dificultad, aunque no los explicitan. Si bien cada teórico estima otras categorías de comprensión lectora, todos concuerdan en que hay cierta complejidad en el interior de cada proceso (Avendaño, Martínez, 2013; Coromoto, Gutiérrez, 2012; Franco et al., 2016; Johnston et al., 2008; Martínez-Díaz et al., 2011; Saulés, 2012; Solé, 2012) y que el desarrollo de la comprensión lectora supone la capacidad de los aprendices para lograrlos todos.

Es claro que para el estudiante cada proceso es complejo en el manejo de la información. Al respecto, los resultados de los estudiantes mexicanos de nivel secundaria en los procesos de interpretación y análisis de la prueba PISA no son alentadores (Herrera, López, Rodríguez, Petit, 2017; OCDE, 2016a). Por lo tanto, existen algunas consideraciones relacionadas con la interpretación y el análisis de la información de un texto. Para la $\operatorname{OCDE}(2009,2013)$, interpretar es un proceso que otorga sentido a algo no expresado en el texto; aquí, el lector debe reconocer los significados o divergencias en una parte o el texto completo, aun cuando no se expliciten; además, puede limitarse a la identificación de un tema o ubicar la idea principal del texto (Saulés, 2012; Solé, 2012). En consecuencia, un estudiante cumple con el proceso de interpretación siguiendo un orden lógico y organizado dentro del texto, infiriendo un contexto o cuando está supeditado al propósito del autor (Lozano, Jiménez, 2011; OCDE, 2009, 2013; Saulés, 2012); también acude, desde a los conocimientos más cercanos a su experiencia hasta reconocer conceptos del lenguaje científico o literario. En contraste, el proceso de mayor dificultad que puede alcanzar un individuo se vincula con el análisis de un texto, el cual incluye la interpretación. Para lograr el análisis, el lector asocia lo leído con sus aprendizajes, conocimientos previos, incluso, aquello ajeno al contexto educativo (OCDE, 2013) que le permitirá evaluar y criticar la información. Ello supone un "alto nivel de capacidad metacognitiva" (Saulés, 2012: 43), es decir, el individuo pondrá en práctica las habilidades para "comparar, contrastar o formular hipótesis" (OCDE, 2013: 70) de la información.

Si bien el grado de complejidad de los diversos procesos cognitivos incide en el logro del educando en comprensión lectora, González et al. (2010) y Solano, Manzanal, Jiménez-Taracido (2016) señalan la existencia de factores exógenos al proceso de tratamiento de la información que influyen en el desempeño de los educandos. Segoviano, Palomo y Cantú (2013) refieren que los factores exógenos son los factores estimuladores del contexto cultural y académico; es decir, "son aspectos que escapan al control del individuo y que no dependen de él" (Romero, Hernández, 2019: 268), entre ellos, destaca el aspecto estructural (contexto social, situación económica de los individuos, estrategias metodológicas) (Moreira, 2009). De los factores exógenos resaltan 
las particularidades o condiciones del entorno donde se desenvuelve el individuo. Por lo tanto, en esta investigación los factores exógenos se vinculan con los elementos académicos y socioculturales a los que se expone el estudiante en la lectura.

Otro de los factores incidentes en la lectura, según Segoviano et al. (2013), son los tipificados como endógenos. Romero y Hernández (2019), Segoviano et al. (2013) y Yilmaz (2009) aseguran que los factores endógenos son los controlados directamente por el individuo, y repercuten en el aspecto relacional (apoyo sociofamiliar) y personal (perfil demográfico, género, edad, capacidades, motivaciones y esfuerzo personal) (Moreira, 2009). A partir de ello, los factores endógenos indagados en este estudio son los personales. Ambos factores (exógenos y endógenos) desempeñan un papel valioso en el logro académico de los estudiantes (Yilmaz, 2009).

Según Franco et al. (2016), estos factores se pueden agrupar en académicos, socioculturales y personales. Siguiendo a este autor, los factores académicos son aspectos o acciones íntimamente ligados al entorno educativo, que pueden influir en el desarrollo escolar de un estudiante. De estos factores, Pearson, Ferdig, Blomeyer y Morán (2005), Avendaño y Martínez (2013), Herrera et al. (2017), Lysenko y Philip (2014) y el National Reading Panel (NRP por sus siglas en inglés) (2000) exploraron la utilización de las TIC para favorecer el desarrollo de la comprensión lectora. Los resultados de estos estudios revelaron interés de los estudiantes al utilizar las TIC en el aula, afirmando que pueden contribuir en el desarrollo de la comprensión lectora.

Brunner y Elacqua (2004) consideran los factores socioculturales como el efecto ocasionado por la familia y los amigos para la mejora del rendimiento académico de los estudiantes, vinculados con el entorno social, cultural y socioeconómico (Franco et al., 2016). Los resultados de Franco et al. (2016) evidencian que un mayor nivel socioeconómico de los estudiantes favorece el desarrollo de la comprensión lectora. Lozano y Jiménez (2011) relacionaron el rendimiento de la comprensión lectora con el nivel educativo de los padres, y encontraron una correlación positiva con el desempeño de los estudiantes en las habilidades lectoras (OCDE, 2003) de la formación secundaria.

Desde otra perspectiva, para Garbanzo (2007) los factores personales son elementos o condiciones inherentes a un individuo, donde las relaciones establecidas con el otro se pueden originar en situaciones intangibles, sociales e institucionales, permitiéndole cierto aprovechamiento en el contexto educativo. Akande y Oyedapo (2018) indagaron la asociación de la comprensión lectora con el tiempo dedicado a la lectura y la motivación. En el primer caso, reportaron que los estudiantes de secundaria deben tener un tiempo específico de lectura con diversos recursos, lo cual podría mejorar su comprensión lectora. Hablando de motivación, carecer de ella limita la comprensión de lo que se enseña en el contexto educativo (Cassany et al., 2003). Por otra parte, Julià (2016), el INEE (2010), Johnsson-Smaragdi, Jönsson (2006), Merisuo-Storm (2006), la OCDE (2013, 2016a), Summers (2013), y el UIS (2017a), estudiaron la relación entre la 
comprensión lectora y el género, encontrando mejores resultados en el aprovechamiento de las mujeres que en los hombres. Sin embargo, Vlachos y Papadimitriou (2015) no encontraron influencia del género en la comprensión lectora de los estudiantes. En otra vertiente, Sturm (2003), Vlachos y Papadimitriou (2015) analizaron el nexo entre comprensión lectora y edad, demostrando un efecto sustancial vinculando la edad con el rendimiento de la lectura. Finalmente, Herrera et al. (2017) analizaron el interés del estudiante hacia la lectura, pero los resultados no fueron óptimos, lo cual afecta en el proceso de comprensión lectora de los pupilos.

Nergis (2013) resalta la necesidad de indagar más sobre la asociación entre la comprensión lectora y los factores influyentes. A la fecha, los factores socioculturales, académicos y personales han sido motivo de estudios al indagar los factores exógenos y endógenos que afectan los hábitos de lectura. No obstante, es importante señalar la necesidad de estudios que busquen articular estos factores con los niveles de comprensión lectora que demuestran los estudiantes en sus niveles educativos (Solano et al., 2016; Vlachos, Papadimitriou, 2015). En virtud de los resultados desalentadores de los estudiantes respecto a la interpretación y análisis de los textos en pruebas como PISA, se requieren entonces investigaciones para conocer los factores académicos, socioculturales y personales que podrían asociarse al bajo o alto rendimiento de los alumnos de secundaria, relacionados con estos procesos. En 2015, los datos de la prueba PISA demostraron que los procesos de interpretación y análisis presentan mayor complejidad, comparados con los procesos de extraer y recuperar. La prueba PISA hace una valoración del aprovechamiento de la comprensión lectora en puntajes; la puntuación máxima alcanzada en ese año fue de 800 puntos. En esta prueba, México logró 423 puntos (INEE, 2017; OCDE, 2016d). Partiendo de los resultados que obtienen los educandos, este organismo determina tres niveles: el inferior, de 262 a 335 puntos, el intermedio de 407 a 480 puntos, y el nivel superior, de 626 puntos en adelante (INEE, 2017). Con base en esta escala de puntajes, de los 7,568 alumnos mexicanos evaluados en lectura durante la prueba PISA 2015, 41\% alcanzó nivel inferior, 54\% el intermedio, y el nivel más complejo fue logrado por $5 \%$ de los estudiantes de secundaria (INEE, 2017). En consecuencia, a partir de los datos mostrados, los alumnos mexicanos se ubican en el nivel intermedio del logro en lectura, con 54 por ciento. Así, estos estudiantes son capaces de localizar la información de un texto, reconocer la relación entre varias partes del texto, comparar o contrastar ideas, reconocer la idea principal, cotejar, diferenciar o clasificar y construir el significado de una palabra (OCDE, 2016d). Sin embargo, los resultados indican que estos estudiantes no son capaces de lograr los procesos de interpretación y análisis.

A partir de las consideraciones presentadas en las secciones anteriores, el objetivo de esta investigación fue indagar los factores académicos, socioculturales y personales de los estudiantes de secundaria en el sureste mexicano, con dos perfiles diferentes en su comprensión lectora: 1) aquellos que logran resultados óptimos en los procesos de interpretación y análisis de la información, y 2) aquellos que muestran un perfil endeble en estos niveles de comprensión lectora.

Diólopos sobre Educación año 12 | número 23 | julio-diciembre 2021 | ISSN 2007-2171 


\section{Método y materiales \\ Diseño del estudio}

Para lograr su objetivo, este estudio adoptó un enfoque cuantitativo, con diseño descriptivo-correlacional (Cohen, Manion, Morrison, 2007). Este diseño permite hacer inferencias empleando datos numéricos para lograr contribuir en la generalización de los resultados. Específicamente, mediante el diseño descriptivo-correlacional, no solo se realizará la descripción de los factores personales, socioculturales y académicos que caracterizan el proceso de comprensión lectora, sino que además se podrá valorar su posible relación con el perfil de comprensión lectora de los estudiantes.

\section{Contexto y participantes}

Para la selección de los participantes se acudió al muestreo no probabilístico por conveniencia (Cohen et al., 2007). Con base en esta consideración metodológica, la investigación se realizó en una escuela secundaria pública ubicada en una zona urbana del centro de la ciudad de Villahermosa, en el estado mexicano de Tabasco. Su población fue de 677 estudiantes, de los cuales 490 cursaban el turno matutino y 187 el vespertino. Se seleccionó esta institución debido a los resultados presentados por la Secretaría de Educación Pública (SEP) en la prueba Planea 2017. En el apartado de comprensión lectora, de cada 10 alumnos, dos alcanzaron el proceso de interpretación, y cuatro lograron el proceso de análisis (SEP, 2017), lo cual es sustantivo de tomar en cuenta para esta pesquisa.

Se seleccionaron participantes que cursaban la asignatura de español en el tercer año; esto es esencial puesto que, en este grado, los estudiantes ya deben contar con los aprendizajes clave de la asignatura de español (INEE, 2016, 2018; SEP, 2016), y haber desarrollado las habilidades de comprensión lectora necesarias. Por ende, deberían ser capaces de resolver una prueba que evalúe los procesos de interpretación y análisis en la comprensión lectora. Inicialmente participaron 164 estudiantes; no obstante, algunos no lograron responder los dos instrumentos aplicados en el estudio. Por lo tanto, solo se retuvieron 151 alumnos que completaron todas las herramientas administradas en la indagación. Las características sociodemográficas se presentan en el apartado de resultados, en virtud de constituir un factor de interés de la investigación.

\section{Instrumentos de recolección de datos}

Para obtener la información de este estudio se emplearon dos instrumentos: la prueba Planea de los años 2016 y 2017, y una encuesta (Cohen et al., 2007; Dörney, 2002; Johnson, 1959).

Prueba Planea. Para valorar la habilidad lectora de los estudiantes se realizó la adaptación de la prueba Planea de los años 2016 y 2017. Esto permitió explorar los procesos de interpretación y análisis con una escala de intervalo (Glass, Hopkins, 1996). En la adaptación de la prueba, 12 
reactivos indagan el análisis de contenido y cuatro el desarrollo de una interpretación, haciendo un total de 16 preguntas que evalúan la comprensión lectora. Estas son de opción múltiple (Boyle, Fisher, 2007; Cohen et al., 2007, Crocker, Schmitt, 1987; Erguven, 2003), con cuatro opciones de respuesta, donde solo una opción es la correcta. Así, las respuestas correctas con respecto a cada tipo de proceso lector se adicionan para obtener un puntaje máximo de cuatro en interpretación y 12 en el análisis de un texto; dentro de esas opciones, se encuentran aleatoriamente los distractores (INEE, 2018). Para asegurar la confiabilidad durante la recolección de respuestas se emplearon Pruebas Paralelas (Boyle, Fisher, 2007; Cohen et al., 2007), las cuales consistieron en una versión A y una versión B de las pruebas 2016 y 2017. En estas versiones se incluyeron los mismos reactivos pero en diferente orden. En consecuencia, se suministraron cuatro pruebas simultáneamente (versiones 2016A, 2016B, 2017A, y 2017B) a distintos alumnos de la muestra. La duración de la prueba fue de 40 minutos (Boyle, Fisher, 2007). Para asegurar la confiabilidad en la codificación de los datos en la adaptación de la prueba Planea 2016 y 2017, se acudió al Inter Rater Reliability (Boyle, Fisher, 2007; Cohen et al., 2007), que consiste en contabilizar los resultados logrados por cada estudiante para crear la base de datos. Después de dos semanas, las pruebas fueron verificadas una vez más, de manera descendente, para confirmar si las codificaciones asignadas habían sido acertadas; no obstante, se observó que durante la codificación inicial los datos habían sufrido alteraciones, mismas que en la segunda revisión fueron subsanadas.

Encuesta. Para identificar los factores académicos, socioculturales y personales que contextualizan la situación lectora de los estudiantes dentro y fuera del aula, se aplicó una encuesta estructurada (Cohen et al., 2007; Dörney, 2003; Johnson, 1959), adaptándola, del Ministerio de Educación, Cultura y Deporte de España ([MECDE] 2001), que incluía 77 preguntas. Como muestra la tabla 1, las preguntas exploraban los factores académicos $(n=11)$, factores socioculturales $(n=$ 22) y los factores personales $(n=44)$. Estas preguntas se agruparon en cuatro grandes secciones de la encuesta que se explican a continuación. 
Tabla 1. Distribución de preguntas por factores que inciden en la lectura, según las secciones de la encuesta

\begin{tabular}{|c|c|c|c|c|c|c|}
\hline Secciones & Factores acadé & $\cos$ & $\begin{array}{l}\text { Factores socio } \\
\text { rales }\end{array}$ & ultu- & Factores persor & iales \\
\hline & Preguntas & Total & Preguntas & Total & Preguntas & Total \\
\hline $\begin{array}{l}\text { Sección I } \\
\text { Perfil del encuestado }\end{array}$ & & 0 & $1,2,3,4,5$ & 5 & & 0 \\
\hline $\begin{array}{l}\text { Sección II } \\
\text { Contextualizando la } \\
\text { lectura }\end{array}$ & $30,36,37$ & 3 & $\begin{array}{l}6,7,19,22, \\
27,31,32,33, \\
34\end{array}$ & 9 & $\begin{array}{l}8,9,10,11, \\
12,13,14,15, \\
16,17,18,20, \\
21,23,24,25, \\
26,28,29, \\
35,38,39,40, \\
41\end{array}$ & 24 \\
\hline $\begin{array}{l}\text { Sección III } \\
\text { Tecnologías de la } \\
\text { Información y la Co- } \\
\text { municación }\end{array}$ & 45,50 & 2 & $42,48,49$ & 3 & $43,44,46,47$ & 4 \\
\hline $\begin{array}{l}\text { Sección IV } \\
\text { Estrategias de lectura }\end{array}$ & $\begin{array}{l}54,69,71,73, \\
74,75\end{array}$ & 6 & $\begin{array}{l}51,52,53 \\
55,77\end{array}$ & 5 & $\begin{array}{l}56,57,58,59 \\
60,61,62,63 \\
64,65,66,67 \\
68,70,72,76\end{array}$ & 16 \\
\hline TOTAL & & 11 & & 22 & & 44 \\
\hline
\end{tabular}

La sección I, conformada por cinco preguntas, exploró el perfil del encuestado. La segunda sección indagó el contexto de la lectura, e incluyó 36 cuestionamientos. La III investigó las tecnologías de la información y la comunicación, con nueve aspectos relativos a ella. La última sección abordó las estrategias de lectura; los participantes respondieron 27 interrogantes. En las secciones I, II y IV se incluyeron preguntas dicotómicas (Cohen et al., 2007), un ejemplo de ellas fue el sexo: hombre o mujer. También se adicionaron preguntas de opción múltiple (Cohen et al., 2007), como el grado máximo de estudio de los padres; las opciones fueron: sin estudios, primaria, secundaria, técnico, bachillerato y estudios universitarios. El total de las preguntas contenidas en la encuesta fue de 77. En el interior de cada sección se exploraron los factores personales, socioculturales y académicos. Para el propósito del análisis en este estudio, las preguntas fueron agrupadas por factores, no por secciones. Estas se contabilizaron como escala nominal (Glass, Hopkins, 1996). Así, se determinó la frecuencia de participantes que seleccionan una opción de respuesta. Para garantizar la confiabilidad en el diseño del instrumento se acudió a las Formas de Pruebas Paralelas (Boyle, Fisher, 2007; Cohen et al., 2007); es decir, en ambas versiones se mantuvieron las cuatro dimensiones establecidas; sin embargo, en el interior de 
las versiones se cambió el orden de cada sección, respetándose el orden de las preguntas. La confiabilidad en la codificación de los datos en la encuesta se consideró a partir del Inter Rater Reliability (Boyle, Fisher, 2007; Cohen et al., 2007), este fue realizado numerando las encuestas de 1 a 151; posteriormente, se creó la base de datos con los resultados obtenidos. Pasadas dos semanas, se revisaron nuevamente las encuestas en orden descendente, verificando si se había codificado correctamente; sin embargo, los resultados presentaron incongruencias y se corrigieron.

Para asegurar la validez de contenido y constructo de la encuesta, se acudió a la valoración de un grupo de expertos (Boyle, Fisher, 2007; Cohen et al., 2007) en psicología y educación. Posterior a sus sugerencias, y debido a la opacidad semántica de las preguntas, se adecuaron dos preguntas de la sección I, una de la sección II, y una correspondiente al segmento IV. En esta última sección se descartaron tres interrogantes por estar duplicadas, y se añadieron tres peguntas para lograr el objetivo del instrumento.

\section{Resultados}

En los siguientes apartados, primero se describen los resultados de los participantes en la prueba de comprensión lectora y la creación de los grupos de estudiantes con perfil de comprensión lectora óptimo y endeble. Posteriormente, se describen por separado las condiciones académicas, socioculturales y personales que caracterizan a los estudiantes clasificados en cada perfil de comprensión lectora.

\section{Resultados en lectura y clasificación de participantes por condición}

Los resultados en la prueba Planea fueron analizados empleando el programa SPSS Statistics v25. En virtud de que esta es una prueba donde se recogen datos numéricos de intervalo, primero se procedió a verificar la normalidad en la distribución de los datos, con el objetivo de identificar la prueba estadística que posteriormente se requeriría para comparar los resultados obtenidos en cada versión de la prueba. En estos análisis se emplearon los puntajes globales obtenidos por los participantes; es decir, la suma de los resultados de interpretación y análisis. La prueba de Kolmogorov-Smirnov solo reveló normalidad en los resultados de la prueba 2017B, $p$ $=0.13$. Por lo tanto, para verificar la igualdad de respuestas entre las versiones A y B se empleó la prueba no paramétrica de U-MannWhitney. Los resultados revelaron similitud en los resultados entre la prueba 2016A $(n=41 ; M=40.11)$ y $2016 \mathrm{~B}(n=40 ; M=41.91), U=477.5, p=0.73$, y las versiones 2017A $(n=42 ; M=32.87)$ y $2016 \mathrm{~B}(n=28 ; M=39.45), U=1448.5, p=0.19$. En consecuencia, a continuación, no se hace diferencia entre pruebas.

Para definir la condición en comprensión lectora, se identificaron los estudiantes como a) con perfil óptimo o b) endeble. Esta identificación se operacionalizó distinguiendo, mediante valores $Z$, aquellos estudiantes que tenían altos y bajos puntajes en la prueba Planea con res-

Diólo pos 
pecto a la media grupal. En consecuencia, los puntajes globales obtenidos en esta prueba se transformaron en valores $Z$ (Field, 2013; Glass, Hopkins, 1995; Larson-Hall, 2010; Muijs, 2004), tomando como referencia la media grupal $(M=9)$ y la desviación estándar ( $D S=3.22)$. Los estudiantes con un valor $Z$ menor a 1 se clasificaron con perfil endeble, y aquellos con un valor $Z$ mayor a 1 se identifican con perfil óptimo. Desde este análisis, de los 151 estudiantes $19 \%$ se agruparon en condición de perfil óptimo $(n=29 ; M=13.6, D S=.85)$ y $26 \%$ con perfil endeble $(n=40 ; M=$ $5 ; D S=1.29$ ).

\section{Factores académicos de los estudiantes con perfil endeble}

Para identificar patrones de respuesta vinculadas a los factores académicos en las encuestas de los estudiantes con perfil de lectura endeble, se emplearon análisis de frecuencia. Estos análisis muestran los siguientes resultados con respecto a los factores académicos explorados en las preguntas distribuidas en las diferentes secciones de la encuesta (véase tabla 1). Para $62.5 \%$ de los estudiantes con perfil de lectura endeble, la lectura les ayuda a entender mejor las clases. A la mayoría de los participantes, los profesores les recomiendan libros (72.5\%), los motivan a leer (70\%) y cuando no comprenden un texto, les disipan sus dudas (57.5\%). No obstante, $57.5 \%$ de los estudiantes no realizan las lecturas sugeridas por sus profesores. Respecto al uso del internet, $75 \%$ de los estudiantes coinciden que les favorece en la comprensión lectora, y a $97.5 \%$ les ayuda en sus tareas.

\section{Factores socioculturales de los estudiantes con perfil endeble}

Con respecto a las preguntas relacionadas al factor sociocultural (véase tabla 1), los análisis de frecuencia revelaron que la mayoría de los alumnos con perfil endeble cuenta con una edad que fluctúa entre 14 (52.5\%) y 15 años (40\%). Predominan los hombres (72.5\%). La mayoría de los estudiantes (67.5\%) viven con ambos padres, quienes frecuentemente cuentan con estudios universitarios; específicamente, se observa que $62.5 \%$ de las madres y $60 \%$ de los padres tienen formación profesional. Muchos de estos estudiantes (55\%) tienen acceso a los libros en casa. El mes anterior a la encuesta, $70 \%$ había leído por gusto más de un libro. Resulta interesante la opinión de estos participantes, quienes consideran que la lectura les enseña a expresarse mejor $(85 \%)$, y les da reconocimiento ante otras personas (57.5\%). Sin embargo, en su casa, gran parte de ellos no leen el periódico (55\%), ni revistas (60\%), y tampoco han tomado asesorías para mejorar su lectura (80\%). Generalmente, los estudiantes con perfil endeble comentan la lectura con sus amigos y otras personas (57.5\%). Además, cuentan con un amigo a quien le gusta leer (52.5\%), pero $75 \%$ de los participantes no lee cuando su mejor amigo lee. Si los participantes no comprenden un texto, mayormente recurren a su mejor amigo (52.5\%) o a su madre (55\%) para aclarar dudas, en lugar de acudir al padre (65\%) o hermano (80\%). Atendiendo a las TIC, la 
mayoría (97.5\%) tiene internet en casa; su uso les permite acceder a blogs (80\%) y a las redes sociales $(97.5 \%)$.

\section{Factores personales de los estudiantes con perfil de lectura endeble}

Los análisis de frecuencia en las respuestas de los estudiantes con perfil lector endeble, vinculados a los factores personales (véase tabla 1), muestran las siguientes condiciones. A la mayoría (72.5\%) les gusta leer y prefiere un libro impreso (52.5\%); leen de una a dos veces por semana $(57.5 \%)$ y de una a tres horas (82.5\%). Aunque les gusta leer cuentos (55\%), libros ilustrados (62.5\%), cómics (67.5\%) e historietas (67.5\%), otros no leen novelas (52.5\%), revistas (62.5\%) ni los mangas (70\%), y 50\% lee el periódico. Cuando los estudiantes pretenden leer un libro, mayormente leen el título (82.5\%), la introducción (72.5\%), o el final (52.5\%); 70\% lee por recomendación, y otros (70\%) consideran una portada atractiva. En contraste, muchos alumnos niegan leer sobre el autor (57.5\%) o definir el propósito de la lectura (55\%).

De los encuestados, $60 \%$ se sienten motivados a leer por sus padres, hermanos, tíos o abuelos, aunque la razón principal de su lectura es por gusto y para aprender (65\%). El 55\% de los participantes usualmente lee en voz alta, otros (55\%) contextualizan las palabras desconocidas para entender el texto, $87.5 \%$ aprende el significado de estas y $70 \%$ de los participantes analiza los textos. Casi todos (92.5\%) indicaron que con la lectura aprenden mucho; a más de la mitad les hace sentir bien (77.5\%), otros, al realizar la lectura emplean su imaginación (87.5\%). Como parte de las estrategias de lectura empleadas, $87.5 \%$ de los participantes, al no comprender un texto lo lee nuevamente para entenderlo; $55 \%$ lee sin pensar en el contenido que va a encontrar, y la mayoría (70\%) predice de lo que va a tratar el autor. 70\% hace pausas y reflexiona si comprende o no lo que lee; $72.5 \%$ conecta las ideas con situaciones reales, y gran parte de los participantes (85\%) imagina la historia. 55\% realiza la lectura pensando en el contenido que va leyendo, y $65 \%$ concluye la lectura pensando en el contenido leído.

La encuesta provee resultados inesperados en los estudiantes con perfil endeble respecto a sus hábitos lectores. Cuando leen por placer emplean diversas estrategias académicas. En este caso, los participantes subrayan ideas principales (45\%), construyen una historieta (27.5\%), redactan una síntesis (25\%), hacen resumen (37.5\%), mapa conceptual (20\%), cuadro sinóptico (7.5\%) o elaboran inferencias (40\%). En relación con la tecnología, $77.5 \%$ utiliza computadora y $95 \%$ busca información en el celular para enriquecer su comprensión durante la lectura. El uso del internet permite a los participantes leer un libro (75\%), y aprender cosas nuevas (95\%).

\section{Factores académicos de los estudiantes con perfil de lectura óptimo}

Los análisis de frecuencia de las respuestas relacionadas con los factores académicos en los estudiantes con perfil de lectura óptimo (véase tabla 1) revelaron las siguientes condiciones. A $58.6 \%$ de los participantes la lectura les ayuda a entender mejor las clases. Un porcentaje mayor 
(69\%) dijo que los profesores les recomiendan libros; sin embargo, 51.7\% de los estudiantes no los lee. $82.8 \%$ son motivados por sus profesores para que lean, y al no comprender el texto (69\%), también los orientan y aclaran sus dudas. Respecto a la utilización del internet, a 69\% de los estudiantes les permite mejorar su comprensión lectora y a todos (100\%) les ayuda en tareas.

\section{Factores socioculturales de los estudiantes con perfil de lectura óptimo}

En la exploración de respuestas de los educandos con un perfil lector óptimo, asociadas a los factores socioculturales (véase tabla 1), los análisis de frecuencia muestran que los participantes corresponden mayormente (55.2\%) al género masculino, con edades entre 14 (51.7\%) y 15 años (48.3\%). La mayoría (55.2\%) vive con ambos padres, quienes generalmente son profesionistas; se observa que $62.1 \%$ de las madres y $65.5 \%$ de los padres tienen estudios universitarios. Gran parte de los estudiantes leen el periódico en casa (55.2\%), lugar donde mayormente (65.5\%) acceden a libros; sin embargo, $51.7 \%$ de los participantes no lee revistas en casa y $79.3 \%$ no ha tomado asesorías extraescolares para optimizar su lectura. Un mes previo a la encuesta, 75.8\% de los estudiantes había leído por gusto más de un libro. 93.1\% de los participantes mostró una situación extraordinaria al puntualizar que leer les enseña a expresarse mejor, pero $58.6 \%$ considera no darles reconocimiento ante otras personas. Habitualmente, los participantes con desempeño óptimo comentan la lectura con sus amigos y otras personas (75.9\%). 55.2\% de los estudiantes enfatizó que a su mejor amigo le gusta leer, en cambio, 58.6\% no lee cuando su mejor amigo lee. Al no comprender un texto, 58.6\% de los estudiantes acude a su mejor amigo o a su mamá (55.2\%) para aclarar dudas, pero no recurren al papá (72.4\%) o al hermano (72.4\%). Relativo a la tecnología, la mayoría tiene internet en casa (96.6\%), 79.3\% lo usa para acceder a blogs y todos (100\%) a las redes sociales.

\section{Factores personales de los estudiantes con perfil de lectura óptimo}

Correspondiente a los análisis de frecuencia en las respuestas de los factores personales (véase tabla 1) de los alumnos con desempeño óptimo, se derivaron las siguientes tendencias. A la mayoría (86.2\%) les gusta leer y 58.6\% prefiere leer un libro impreso. Gran parte lee de una a dos veces a la semana (58.6\%), y 72.4\% dedica de una a tres horas. Los estudiantes prefieren leer historietas (51.7\%), cuentos (62.1\%), cómics (65.5\%) y libros ilustrados (75.9\%), no así novelas (51.7\%), revistas (58.6\%), mangas (69\%) o el periódico (75.9\%).

$72.2 \%$ de los estudiantes son motivados a leer por sus padres, hermanos, tíos, abuelos, u otro familiar. Sin embargo, la razón principal de lectura es por gusto (51.7\%). Cuando los estudiantes leen por gusto, $65.5 \%$ analiza los textos, la mayoría (86.2\%) aprende mucho; a otros más, leer les hace sentir bien (89.7\%) y a un porcentaje mayor (93.1\%) les ayuda a imaginar cosas o situaciones. $65.5 \%$ de los participantes contextualiza las palabras desconocidas y $72.4 \%$ aprende su significado. $51.7 \%$ lee sin pensar en el contenido que va a encontrar, otros (69\%) tratan de 
predecir el contenido del texto y $65.5 \%$ lee pensando en el contenido que va leyendo. $65.5 \%$ de los estudiantes hace pausas y reflexiona si comprende o no lo que lee; sin embargo, 93.1\%, al no comprender un texto, lo lee nuevamente y trata de comprenderlo. 79.3\% conecta las ideas con situaciones reales, la mayoría imagina la historia (89.7\%), 82\% termina la lectura pensando en el contenido leído y normalmente los participantes no leen en voz alta (51.7\%).

Para los participantes con perfil óptimo, lo más relevante al leer un libro es la introducción (62.1\%), el título (82.8\%), una portada atractiva (62.1\%) o leer por recomendación (75.9\%); contrariamente, $72.4 \%$ no lee el final o sobre el autor (62.1\%), y nunca definen el propósito de la lectura (72.4\%). Es indispensable mencionar que algunos estudiantes con un perfil óptimo lector emplean diversas estrategias académicas cuando leen por placer; por ejemplo, elaboran un resumen (6.9\%), un mapa conceptual (6.9\%) o un cuadro sinóptico (13.8\%). El 27.6\% subraya ideas principales, sintetiza (13.8\%), hace una historieta (3.4\%) o inferencias (27.6\%). Bajo la perspectiva tecnológica, $86.2 \%$ usa computadora y todos los alumnos (100\%) buscan información con su celular. $82.8 \%$ de los participantes usa el internet para leer un libro, y un porcentaje mayor (96.6\%) para aprender cosas nuevas. Finalmente, 55.2\% prefiere las asignaturas de matemáticas y educación física en comparación de otras disciplinas.

\section{Discusión}

Para una mejor comprensión de las condiciones de lectura en estudiantes adolescentes de educación pública, se investigaron los factores académicos, socioculturales y personales en alumnos de nivel secundaria. Estos factores se exploraron tomando en cuenta dos perfiles en la capacidad lectora de los estudiantes: a) perfil óptimo, y b) perfil endeble. En este tenor, a continuación, primero se discuten las similitudes documentadas en los estudiantes con esos perfiles. Posteriormente, se discuten las diferencias entre los grupos de estudiantes.

La motivación a la lectura permite crear en los educandos un deseo de cultivarse (Avendaño, Martínez, 2013). En lo que respecta a los factores académicos, nuestros resultados concuerdan con los presentados por Herrera et al. (2017), y Vlachos y Papadimitriou (2015), donde los profesores motivan a los participantes a leer desde la escuela. En este sentido se observa que, independientemente del perfil lector de los estudiantes, el entorno educativo es pieza clave en el fortalecimiento de sus habilidades lectoras. Sin duda, el apoyo del profesor es cardinal en el aula, ya que los orienta cuando no comprenden un texto y disipa sus dudas, tal vez, por la convivencia diaria en el contexto educativo y la confianza que el profesor les genera. No obstante, los libros recomendados por el docente no son de interés para los estudiantes. En consecuencia, los profesores deberían sugerir textos propios de la edad o de su interés, para lograr su cometido y no limitarse a textos académicos (Akande, Oyedapo, 2018) o los determinados por las instituciones educativas.

Diólopos 
La lectura es el cimiento de la formación académica (Akande, Oyedapo, 2018), y para los estudiantes de la educación elemental y secundaria no es la excepción. Una situación relevante, reportada en esta investigación respecto a los factores personales, es que independientemente de su condición de lectura a los estudiantes les gusta leer, esto coincide con reportes internacionales (Avendaño, Martínez, 2013; Merisuo-Storm, 2006; Summers, 2013). En el contexto estudiado, los participantes prefieren leer un libro impreso, cuentos, cómics, historietas y libros ilustrados (véase también Avendaño, Martínez, 2013; Merisuo-Storm, 2006; Summers, 2013), pero no les gusta leer novelas ni revistas, como en el estudio de Avendaño y Martínez (2013). Un dato notable es que a los participantes no les gusta leer mangas; esto resulta de interés para próximos estudios puesto que actualmente los mangas se han convertido en moda para los jóvenes de secundaria.

Un aspecto interesante en nuestros resultados es que los reportes sugieren que la lectura hace sentir bien a los estudiantes y los estimula a entrar en procesos cognoscitivos donde está activa la imaginación y la creatividad (Johnsson-Smaragdi, Jönsson, 2006). Los participantes contextualizan las palabras desconocidas para entender el texto, y al mismo tiempo aprenden su significado. Leen sin pensar en el contenido que van a encontrar, pero al ir leyendo tratan de predecir de lo que va a tratar el texto. Al no comprender un texto, lo vuelven a leer y tratan de comprenderlo. Conectan las ideas con situaciones reales e imaginan la historia. Leen pensando en el contenido que van leyendo. Además, terminan la lectura pensando en el contenido leído. Estos resultados no pudieron ser contrastados con otros estudios, por lo que se sugiere realizar investigaciones que documenten estas condiciones lectoras para que desde el contexto educativo se favorezcan las estrategias lectoras de los estudiantes. Solé (1996:177) refiere que "las estrategias de lectura aprendidas en contextos significativos contribuyen a la consecución de la finalidad general de la educación que consiste en que los alumnos aprendan a aprender".

Otra información valiosa es la utilización de estrategias académicas usadas por los estudiantes cuando leen por placer, es su vínculo en la construcción de resúmenes y organizadores gráficos que son un apoyo fundamental para favorecer la comprensión lectora (Nergis, 2013). También recurren a subrayar ideas principales, sintetizar, inferir y hacer una historieta, como en el reporte de Avendaño y Martínez (2013) y Franco et al. (2016). En efecto, estos aspectos son determinantes en el proceso lector de los estudiantes y es necesario que las instituciones educativas los estimulen (Lozano, Jiménez, 2011). Los estudiantes ratificaron leer una a dos veces por semana, y de una a tres horas (véase también Johnsson-Smaragdi, Jönsson, 2006).

En lo que respecta a los factores socioculturales, se observa que las personas que interactúan cotidianamente en el contexto social con los estudiantes tienen un impacto sustantivo en el desarrollo lector de los jóvenes. Por ejemplo, los principales motivadores de la lectura en el contexto familiar son los padres, hermanos, tíos y abuelos (véase también Franco, 2016; Lozano, Jiménez, 2011). Esta motivación podría adjudicarse al hecho de que los participantes tienen 
edades entre 14 y 15 años, y la mayoría viven con ambos padres. Sin duda, vivir en un entorno familiar donde los padres influyen directamente en la educación de los jóvenes podría ser el parteaguas para el favorecimiento de la lectura, así como se presenta en Avendaño y Martínez (2013). Adicional a ello, otro aspecto de interés en el desarrollo lector de los estudiantes es que los padres tienen estudios universitarios; caso contrario se muestra en el reporte de Avendaño y Martínez (2013), donde los padres de familia solo alcanzaron los estudios de secundaria. El nivel educativo de los padres de la población estudiada podría ser un indicador para que los jóvenes se vean más motivados en su interés por la lectura. Estos resultados confirman el argumento de Johnsson-Smaragdi y Jönsson (2006), Julià (2016), Vlachos y Papadimitriou (2015), indicando que el núcleo familiar es fundamental para favorecer la lectura e influir positivamente en la comprensión lectora de los hijos.

Por otra parte, conviene resaltar que en la actualidad las tecnologías desempeñan un papel importante en el proceso de aprendizaje de los jóvenes. En nuestro estudio, los resultados indican que las posibilidades de acceso a internet que los estudiantes tienen en sus hogares les permite aprovechar los beneficios inherentes. Por lo tanto, cuando hacen uso de los recursos tecnológicos a su alcance les gusta leer (Avendaño, Martínez, 2013); además, el uso de las TIC les permite que su aprendizaje se favorezca con cosas nuevas. Avendaño y Martínez (2013), Dreyer y Nel (2003) y Johnson, Archibald y Tenenbaum (2010) aseguran que la utilización de las tecnologías estimula los conocimientos de los estudiantes y favorece el aprendizaje (Rusmanayanti, Hanafi, 2018; Wibisono, 2019). De ahí, con el uso del internet los estudiantes no solo pueden tomar a la tecnología como una oportunidad de aprendizaje; los jóvenes aseguraron que esto les ofrece una oportunidad de socialización cuando acceden a redes sociales y a blogs, así como en el estudio de Summers (2013).

Si bien las estrategias de lectura tradicionales benefician en el logro de la comprensión lectora, los resultados de nuestro estudio indican que la tecnología también favorece la lectura, ya que los estudiantes recurren al teléfono celular para buscar información; este hallazgo es consistente con los aportes de Rusmanayanti y Hanafi (2018), y Wibisono (2019). Al respecto, Rusmanayanti y Hanafi (2018), Pearson et al. (2005) y Wibisono (2019) afirman que las herramientas tecnológicas potencian el aprendizaje y comprensión lectora de los estudiantes de secundaria; por ende, las instituciones educativas deberían considerar su inclusión en los programas de estudio como apoyo en sus tareas, así lo aseguran los participantes.

Aunque existe una cantidad importante de estudios en diversos contextos nacionales e internacionales que examinan los factores académicos, socioculturales y sociales de los estudiantes (Brunner, Elacqua, 2004; Franco et al., 2016; Garbanzo, 2007; Herrera et al., 2017; Lozano, Jiménez, 2011; Moreira, 2009; Nergis, 2013; Segoviano et al., 2013; Yilmaz, 2009), no se pueden establecer comparaciones directas, porque muestran resultados de todos los factores sin tomar en consideración los perfiles lectores de los estudiantes. Para ello se sugieren investigaciones 
futuras orientadas a explorar los factores académicos, socioculturales y personales con estudiantes de perfil óptimo y endeble respecto a la lectura.

En el contexto social, donde los padres influyen positiva y significativamente en el aprendizaje y la lectura, uno de los aspectos que resulta interesante es que los estudiantes están respondiendo de forma distinta respecto a lo que les sirve de estímulo para esa lectura en el contexto sociocultural. En este sentido, se destaca que los estudiantes con aprovechamiento óptimo están siendo motivados a leer, porque en su casa siempre van a encontrar un periódico o porque sus padres compran el periódico. Este resultado coincide con los revelados por Avendaño y Martínez (2013), y Johnsson-Smaragdi y Jönsson (2006).

En contraste, aunque los estudiantes que tienen un perfil lector endeble tienen padres profesionistas, así como los jóvenes con perfil de lectura óptimo no encuentran en su contexto familiar el acceso al periódico. Posiblemente, en ese entorno familiar no exista una cultura de leer el periódico o la posibilidad de comprarlo para leerlo, como en el estudio de Avendaño y Martínez (2013); por lo tanto, ante esa situación de los estudiantes con este perfil lector, no existe un interés evidente de la lectura, esto conlleva que pocos participantes lean el diario. Estos resultados no pudieron ser contrastados con otros estudios enfocados en el perfil lector de los estudiantes, lo que hace necesario realizar investigación en este contexto para que los docentes y los padres de familia, en un trabajo conjunto, favorezcan la comprensión de lectura de los jóvenes, y así lograr una educación de calidad (Avendaño, Martínez, 2013; Franco et al., 2016). Es imprescindible que los estudiantes cuenten con el apoyo familiar para su desempeño lector, en caso contrario, si los pilares de la familia se ausentan, evidentemente no ayuda al desarrollo de la comprensión lectora; en consecuencia, repercute de forma negativa en su aprovechamiento académico (Franco et al., 2016). La lectura no solo es un instrumento primordial para la formación académica, sino para que el individuo continúe cultivándose a través del tiempo (Delors, 1998).

Por otra parte, en los resultados de este estudio existe una diferencia marcada respecto a la apreciación de la lectura entre los jóvenes con perfil óptimo y los participantes con perfil endeble. Esa diferencia es que los primeros ven la lectura como una herramienta que les ayuda a mejorar su expresión, lo que concuerda con el argumento de que, al leer, los individuos potencialmente desarrollan sus habilidades comunicativas ampliando su lenguaje y vocabulario (ver Johnsson-Smaragdi, Jönsson, 2006). Sin embargo, para los segundos no es relevante que la lectura los ayude a mejorar; para ellos, lo relevante es que leer les permite crearse una imagen ante los demás o, quizá, les da poder de pertenecer a cierto status dentro de su propio contexto social.

Indistintamente, si para los participantes con perfil endeble la lectura favorece su imagen, es un hecho que la lectura "mejora las relaciones familiares y sociales, estimula la imaginación, nuestros conocimientos y nos acerca a una mejor interpretación del medio en que vivimos" (Sánchez, 2013: 8). 


\section{Conclusión}

Los resultados mostrados en este estudio respecto a los factores académicos, socioculturales y personales son alentadores. Una de las razones se debe a que la población de este estudio realiza actividades de lectura; tal vez esas lecturas no son representativas de los tipos de textos que se esperaría leyeran en el ámbito académico; sin embargo, a pesar de que los estudiantes presentan diferentes perfiles lectores, están leyendo por gusto y están involucrados en la lectura. Los participantes están leyendo en el ámbito familiar mangas, revistas, cómics, el periódico; textos que son acordes a la edad que tienen, pero cuando se exponen al ámbito académico o cuando deben resolver una prueba como la de Planea, sus resultados no presentan resultados favorables. Esto puede deberse a que la lectura realizada no está favoreciendo el desarrollo de los procesos lectores o, en su defecto, porque el tipo de textos mostrados en la prueba o los requeridos por los docentes no son de su interés. Sin duda, el núcleo familiar es la pieza clave mediadora entre los estudiantes y los libros (Franco et al., 2016).

Por otra parte, los resultados también revelan que existen distintas cuestiones que en estudios posteriores deben tenerse en consideración, relacionadas con los factores académicos, socioculturales y personales. Primero se recurrió al muestreo por conveniencia y a una muestra menor a la inicial porque se descartaron estudiantes que no cumplían con las condiciones de comprensión lectora (véase Pearson et al., 2005). A partir de estas condicionantes, es importante reflexionar que, para generalizar nuestros resultados se sugiere, por ejemplo, la realización de estudios comparados en los que se analicen los factores académicos, socioculturales y personales en estudiantes con perfiles endebles y óptimos, pero en contextos educativos más variados que incluyan poblaciones mayores, procedentes de escuelas en zonas marginadas de la ciudad, zonas rurales y urbanas (Akande, Oyedapo, 2018). Si bien los resultados de este estudio, y estudios posteriores, buscan comprender las condiciones que caracterizan a los estudiantes con diferentes niveles de comprensión lectora en pruebas estandarizadas, también es importante que los resultados obtenidos sirvan para emprender estrategias de cambio académico y social, dentro y fuera del aula, para mejorar la calidad en la educación pública.

\section{Referencias}

Akande, S.; R. Oyedapo (2018). Developing the Reading Habits of Secondary School Students in Nigeria: The Way Forward. International Journal of Library Science, 7(1), 15-20. https://doi.org/10.5923/j.library.20180701.03

Avendaño, l.; D. Martínez (2013). Competencia lectora y el uso de las nuevas tecnologías de la información y comunicación. Escenarios, 11(1), 7-22. https://doi.org/10.15665/esc.v11i1.176

Boyle, J.; S. Fisher (2007). Educational Testing. A Competence Based Aproach. Malden: BPS Blackwell. 
Brunner, J.; G. Elacqua (2004). Factores que inciden en una educación efectiva. Evidencia internacional. http://www.opech.cl/bibliografico/evaluacion/BrunnerOEA.pdf

Cassany, D. (2004). Explorando las necesidades actuales de comprensión: Aproximaciones a la comprensión crítica. Lectura y Vida, 25(2), 6-23.

Cassany, D.; M. Luna; G. Sanz (2003). Enseñar lengua. Barcelona: GRAÓ.

Cohen, L.; L. Manion; K. Morrison (2007). Research Methods in Education (6a ed.). Nueva York: Routledge.

Coromoto, M.; O. Gutiérrez (2012). La comprensión de la lectoescritura en la construcción del conocimiento. En la mirada de estudiantes en la educación superior. Congreso Iberoamericano de las Lenguas en la Educación y en la Cultura/IV Congreso Leer.es. Salamanca, España. 5 al 7 de septiembre de 2012.

Crocker, L.; A. Schmitt (1987). Improving Multiple-Choice Test Performance for Examinees with Different Levels of Test Anxiety. The Journal of Experimental Education, 55(4), 201-205.

Delors, J. (1998). La educación encierra un tesoro. Informe de la Comisión de Educación de la UNESCO. UNESCO.

Díaz-Barriga, A. (2015). La prueba PISA 2006. Un análisis de su visión sobre la ciencia. México: UNAM-IISUE.

Dörney, Z. (2002). Questionnaires in Second Language Research. Construction, Administration, and Processing. 2nd Edition. Nueva York: Routledge.

Dreyer, C.; C. Nel (2003). Teaching Reading Strategies and Reading Comprehension within a Technology-enhaced Learning Environment. System, 31, 349-365.

Erguven, M. (2013). Two Approaches to Psychometric Process: Classical Test Theory and Item Response Theory. Journal of Education, 2(2), 23-30.

Field, A. (2013). Discovering Statistics Using IBM SPSS Statistics. Londres: Sage.

Franco, M.; R. Cárdenas; E. Santrich (2016). Factores asociados a la comprensión lectora en estudiantes de noveno grado de Barranquilla. Psicogente, 19(36), 296-310.

Garbanzo, G. (2007). Factores asociados al rendimiento académico en estudiantes universitarios, una reflexión desde la calidad de la educación superior pública. Revista Educación, 31(1), 43-63.

Glass, G.; K. Hopkins (1996). Statistical Methods in Education and Psychology. Pearson Education. González, M.; M. Barba; A. González (2010). La comprensión lectora en educación secundaria. Revista Iberoamericana de Educación, 53(6), 1-11.

Gracida, M. (2012). Los textos continuos: ¿Cómo se leen? La competencia lectora desde PISA. México, INEE.

Hashemifardnia, A.; E. Namaziandost; S. Sajad (2018). The Effect of Implementing Flipped Classrooms on Iranian Junior High School Students' Reading Comprehension. Theory and Practice in Language Studies, 8(6), 665-673. 
Herrera, J.; R. López; Y. Rodríguez; E. Petit (2017). Incidencia de los factores en la comprensión lectora de los estudiantes de décimo grado en la Institución Educativa Distrital Madres Católicas. Escenarios, 15(1), 85-108. http://dx.doi.org/10.15665/esc.v15i1.1123

Institute for Statistics de la UNESCO, UIS (2017a). More than One-half of Children and Adolescents Are not Learning Worldwide. UIS Fact Sheet, 46, 2-26.

http://uis.unesco.org/sites/default/files/documents/fs46-more-than-half-children-notlearning-en-2017.pdf

Institute for Statistics, UNESCO, UIS (2017b). Counting the Number of Children Not Learning Methodology for a Global Composite Indicator for Education. UIS Information Paper, 47, 1-23. http://uis.unesco.org/sites/default/files/documents/ip47-counting-number-children-not-learning-methodology-2017-en.pdf

Instituto Nacional para la Evaluación de la Educación, INEE (2010). México en PISA 2009 (Informe). México: INEE.

Instituto Nacional para la Evaluación de la Educación, INEE (2016). Planea: Una nueva generación de pruebas. México, INEE.

Instituto Nacional para la Evaluación de la Educación, INEE (2017). México en PISA 2015. México: INEE.

Instituto Nacional para la Evaluación de la Educación, INEE (2018). Manual técnico del Plan Nacional para la Evaluación de los Aprendizajes Planea 2015. Sexto de primaria y tercero de secundaria. México: INEE. http://publicaciones.inee.edu.mx/buscadorPub/P1/E/207/P1E207.pdf

Jiménez, E. (2014). Comprensión lectora vs. competencia lectora: qué son y qué relación existe entre ellas. Investigaciones sobre Lectura, (1), 65-74.

Johnson, P. (1959). Development of the Sample Survey as a Scientific Methodology. The Journal of Experimental Education, 27(3), 167-176.

Johnson, T.; T. Archibald; G. Tenenbaum (2010). Individual and Team Annotation Effects on Students' Reading Comprehension Critical Thinking, and Meta-cognitive Skills. Computers in Human Behavior, (26), 1496-1507.

Johnsson-Smaragdi, U.; A. Jönsson (2006). Book Reading in Leisure Time: Long-Term Changes in Young Peoples' Book Reading Habits. Scandinavian Journal of Educational Research, 50(5), 519-540. https://doi.org/10.1080/00313830600953600

Johnston, A.; M. Barnes; A. Desrochers. (2008). Reading Comprehension: Developmental Processes, Individual Differences, and Interventions. Canadian Psychology/Psychologie Canadienne, 49(2), 125-132. https:// https://doi.org/10.1037/0708-5591.49.2.125

Julià, A. (2016). Contexto escolar y desigualdad de género en el rendimiento de comprensión lectora. Revista Española de Investigaciones Sociológicas, (156), 41-58.

https://doi.org/10.5477/cis/reis.156.41 
Kendeou, P.; K. McMaster; T. Christ (2016). Reading Comprehension. Policy Insights from the Behavioral and Brain Sciences, 3(1), 62-69.

Larson, J. (2010). A Guide to Doing Statistics in Second Language Research Using SPSS. Routlege.

Lozano, M.; M. Jiménez (2011). Factores del estudiante y la familia asociados a la comprensión lectora de estudiantes de sexto y noveno grado de educación básica secundaria. Revista Iberoamericana de Psicología: Ciencia y Tecnología, 4(1), 75-87.

Lysenko, L.; A. Philip (2014). Promoting Reading Comprehension with the Use of Technology. Computers \& Education 75, 162-172. https://doi.org/10.1016/j.compedu.2014.01.010

Madero, I.; L. Gómez (2013). El proceso de comprensión lectora en alumnos de tercero de secundaria. Revista Mexicana de Investigación Educativa, 18(56), 113-139.

https://rei.iteso.mx/bitstream/handle/11117/1367/56005.pdf?seque

Márquez, A. (2017). Sobre lectura, hábito lector y sistema educativo. Perfiles Educativos, 39(155), 3-18. https://doi.org/10.22201/iisue.24486167e.2017.155.58100

Martínez-Díaz, E.; N. Díaz; D. Rodríguez (2011). El andamiaje asistido en procesos de comprensión lectora en universitarios. Educación y Educadores, 14(3), 531-555.

Merisuo-Storm, T. (2006). Girls and Boys Like to Read and Write Different Texts. Scandinavian Journal of Educational Research, 50(2), 111-125. https://doi.org/10.1080/00313830600576039

Ministerio de Educación, Cultura y Deporte de España, MECDE (2001). Encuesta sobre hábitos lectores de la población escolar. España.

Ministerio de Educación de Perú (2018). Marco de evaluación de la competencia lectora de PISA 2018. Oficina de Medición de la Calidad de los Aprendizajes.

Monroy, J.; B. Gómez (2009). Comprensión Lectora. REMO, 6(16), 37-42.

Moreira, T. (2009). Factores endógenos y exógenos asociados al rendimiento en matemática: Un análisis multinivel. Revista Educación, 33(2), 61-80.

Muijs, D. (2004). Doing Quantitative Research in Education whit SPSS. Sage Publications.

National Reading Panel, NRP (2000). Teaching Children to Read: An Evidence-based Assessment of the Scientific Research Literature on Reading and its Implications for Reading Instruction.

http://www.nichd.nih.gov/sites/default/files/publications/pubs/nrp/Documents/report.pdf

Nergis, A. (2013). Exploring the Factors that Affect Reading Comprehension of EAP Learners. Journal of English for Academic Purposes, 12, 1-9. https://doi.org/10.1016/j.jeap.2012.09.001

Organización para la Cooperación y el Desarrollo Económicos, OCDE (2003). Informe PISA. Programa para la Evaluación Internacional de Alumnos. Conocimientos y destrezas para la vida. OCDE.

Organización para la Cooperación y el Desarrollo Económicos, OCDE (2009). PISA 2009 Assessment Framework Key Competencies in Reading, Mathematics and Science. OECD.

http://www.oecd.org/pisa/pisaproducts/44455820.pdf 
Organización para la Cooperación y el Desarrollo Económicos, OCDE (2013). PISA 2012 Assessment and Analytical Framework: Mathematics, Reading, Science, Problem Solving and Financial Literacy. OECD Publishing.

Organización para la Cooperación y el Desarrollo Económicos, OCDE (2016a). Dónde se sitúa su centro educativo en el contexto internacional. PISA para centros educativos 2015-2016. Ejemplo de informe de centro España. OCDE.

Organización para la Cooperación y el Desarrollo Económicos, OCDE (2016b). PISA 2015. PISA. Resultados clave. OCDE. http://www.oecd.org/pisa/pisa-2015-Mexico.ESP.pdf

Organización para la Cooperación y el Desarrollo Económicos, OCDE (2016c). Programa para la Evaluación Internacional de Alumnos (PISA) Resultados. México.

http://www.oecd.org/pis/PISA-2015-Mexico-ESP.pdf

Organización para la Cooperación y el Desarrollo Económicos, OCDE (2016d), PISA 2015 Results (Volume I): Excellence and Equity in Education. PISA, OECD Publishing.

https://www.oecd.org/publications/pisa-2015-results-volume-i-9789264266490-en.htm

Organización de las Naciones Unidas para la Educación, la Ciencia y la Cultura, UNESCO (2016). Aportes para la enseñanza de la lectura. OREALC/UNESCO. https://unesdoc.unesco.org/ark:/48223/pf0000244874

Pearson, P.; R. Ferdig; R. Blomeyer; J. Moran (2005). The Effects of Technology on Reading Performance in the Middle-School Grades: A Meta-Analysis with Recommendations for Policy. Learning Point Associates/North Central Regional Educational Laboratory (NCREL).

Pourhosein, A.; N. Banou (2016). How Can Students Improve Their Reading Comprehension Skill? Journal of Studies in Education, 6(2), 229-240.

Reading Study Group, RAND. (2002). Reading for Understanding: Toward an R\&D Program in Reading Comprehension. RAND.

Romero, E.; M. Hernández (2019). Análisis de las causas endógenas y exógenas del abandono escolar temprano: una investigación cualitativa. Educación, 21(22), 263-293.

Rusmanayanti, A.; M. Hanafi (2018). Teaching Reading Comprehension by Using Computer-based Reading: An Experimental Study in Indonesian English Language Teaching. Arab world English. Journal (AWEJ) Special Issue on CALL, (4)

Sánchez, L. (2013). La comprensión lectora: hacia una aproximación sociocultural. Diálogos, 7(12), 7-16.

Saulés, S. (2012). La competencia lectora en PISA. Influencias, innovaciones y desarrollo. México: INEE.

Secretaría de Educación Pública, SEP (2016). Manual para la aplicación, calificación, análisis y uso de los resultados de la prueba. Planea Educación Básica, tercer grado de secundaria, 2016. MéXiCO: SEP-INEE.

Diólo oos 
Secretaría de Educación Pública, SEP (2017). Resultados nacionales. Escuelas secundarias 2017. Bases de datos completa 2017. http://planea.sep.gob.mx/ba/base de datos 2017/

Segoviano, J.; M. Palomo; J. Cantú (2013). Factores endógenos y exógenos que impactan en el uso de la biblioteca en tres universidades del área metropolitana de Monterrey, Nuevo León. Investigación Bibliotecológica, 27(61), 53-81.

Solano, N.; A. Manzanal; L. Jiménez-Taracido (2016). Estrategias de aprendizaje, comprensión lectora y rendimiento académico en educación secundaria. Psicología Escolary Educacional, 20(3), 447-456.

Solé, I. (1996). Estrategias de lectura. Graó.

Solé, I. (2012). Competencia lectora y aprendizaje. Revista Iberoamericana de Educación, 59, 43-61.

Sturm, B. (2003). The Information and Reading Preferences of North Carolina Children. School Library Media Research, 6, 1-30.

Summers, K. (2013). Adult Reading Habits and Preferences in Relation to Gender Differences. Reference \& User Services Quarterly, 52(3), 243-49.

Vlachos, F.; A. Papadimitriou (2015). Effect of Age and Gender on Children's Reading Performance: The Possible Neural Underpinnings. Cogent Psychology, 2(1), 1-10. https://doi.org/10.1080/23311908.2015.1045224

Wibisono, D. (2019). The Effects of Kahoot! In Teaching Reading to Tenth Grade Students. Magister Scientiae, 45, 86-105.

Yilmaz, G. (2009). Exogenous and Endogenous Factors Influencing Students' Performance in Undergraduate Accounting Modules. Accounting Education: An International Journal, 18(1), 51-73. https://doi.org/10.1080/09639280701740142

Zarzosa, L.; M. Martínez (2011). La comprensión lectora en México y su relación con la investigación empírica externa. Revista Mexicana de Psicología Educativa, 2(1), 15-30. 